\title{
Mitochondrial RNA processing in absence of tRNA punctuations in octocorals
}

\author{
Gaurav G. Shimpi ${ }^{1,4^{*}}$, Sergio Vargas ${ }^{1} \mathbb{0}$, Angelo Poliseno ${ }^{1}$ and Gert Wörheide ${ }^{1,2,3^{*}} \mathbb{E}$
}

\begin{abstract}
Background: Mitogenome diversity is staggering among early branching animals with respect to size, gene density, content and order, and number of tRNA genes, especially in cnidarians. This last point is of special interest as tRNA cleavage drives the maturation of mitochondrial mRNAs and is a primary mechanism for mt-RNA processing in animals. Mitochondrial RNA processing in non-bilaterian metazoans, some of which possess a single tRNA gene in their mitogenomes, is essentially unstudied despite its importance in understanding the evolution of mitochondrial transcription in animals.

Results: We characterized the mature mitochondrial mRNA transcripts in a species of the octocoral genus Sinularia (Alcyoniidae: Octocorallia), and defined precise boundaries of transcription units using different molecular methods. Most mt-mRNAs were polycistronic units containing two or three genes and $5^{\prime}$ and/or $3^{\prime}$ untranslated regions of varied length. The octocoral specific, mtDNA-encoded mismatch repair gene, the mtMutS, was found to undergo alternative polyadenylation, and exhibited differential expression of alternate transcripts suggesting a unique regulatory mechanism for this gene. In addition, a long noncoding RNA complementary to the ATP6 gene (IncATP6) potentially involved in antisense regulation was detected.
\end{abstract}

Conclusions: Mt-mRNA processing in octocorals possessing a single mt-tRNA is complex. Considering the variety of mitogenome arrangements known in cnidarians, and in general among non-bilaterian metazoans, our findings provide a first glimpse into the complex mtDNA transcription, mt-mRNA processing, and regulation among early branching animals and represent a first step towards understanding its functional and evolutionary implications.

Keywords: Mitochondrial RNA, Polycistronic transcripts, Alternative polyadenylation (APA), Long noncoding RNA (IncRNA), Mitogenome, Cnidaria, Octocorals, tRNA punctuation, Non-bilaterian

\section{Background}

Two major evolutionary events occurred early in the animal history forging the majority of animals, as we know them today: the origin of their multicellularity, and the origin of bilateral symmetry. Multiple genomic changes accompanied these morphological transitions, and different genome sequencing projects give us a glimpse into these changes $[1,2]$. Undoubtedly, these transitions also correlate with multiple changes in mitochondrial genome (mitogenome) architecture and organization [3].

\footnotetext{
*Correspondence: nature.gaurav@gmail.com; woerheide@lmu.de 1 Department of Earth and Environmental Sciences, Palaeontology \& Geobiology, Ludwig-Maximilians-Universität München, Richard-Wagner Str. 10, 80333 Munich, Germany

Full list of author information is available at the end of the article
}

The metazoan mitochondrial genome underwent reductive evolution, transferring most of its genome content to the nucleus $[4,5]$. The majority of these alterations in mitogenome content include the loss of ribosomal proteins and some tRNA genes, changes in the genetic code, disappearance of introns, and further compaction of mitochondrial DNA (mtDNA). As an aftermath, a quintessential animal mitochondrial genome harbors only 13 genes encoding essential energy pathway proteins, 2 ribosomal RNA genes and 22 transfer RNA genes. This composition is nearly invariable among bilaterians in terms of gene content [6]. However, alterations in mitogenome content, size and organization are more prominent and peculiar among non-bilaterian animals. The mitogenomes of non-bilaterian metazoan phyla 
comprise several novelties compared to the canonical animal mitogenomes [7]. These include, the presence of group I and group II introns in sponges and scleractinians [8-12], additional protein coding genes and/ or unknown ORFs and gene duplications in anthozoans [13-16], linear mitogenomes in calcisponges and medusozoans $[17,18]$, among other. Therefore, as a hotspot of mitochondrial genome diversity, early branching animals present a unique opportunity to understand the evolution of mitochondrial genome architectures as well as the fundamental processes governing its functionality and maintenance.

The significantly reduced but extremely crucial repertoire of genes present in animal mitogenomes is fundamental to its molecular and cellular functions, and to gain a deeper understanding of these processes it is essential to understand the expression and processing of mitochondrial gene transcripts. The majority of information available so far on the mitogenome transcription originates from bilaterian members of the animal kingdom [19-22]. In this regard, the canonical vertebrate mitogenome is known to transcribe symmetrically as polycistronic precursors spanning the entire heavy $(\mathrm{H}-)$ and light (L-) strands [23]. The 22 tRNAs interspersed throughout the mitogenome serve as punctuation marks that are recognized and cleaved at $5^{\prime}$ and $3^{\prime}$ ends by the mitochondrial RNase $\mathrm{P}$ and RNase $\mathrm{Z}$, respectively $[24,25]$. The genes within these precursors are simultaneously liberated for maturation following this mt-tRNA processing step. Consequently, in most bilaterian metazoans, most mature mitochondrial mRNAs are monocistronic, with ATP8-ATP6 and ND4L-ND4, which are known to exist as bicistronic elements, as the only exceptions. Finally, all messengers end with the post-transcriptional addition of 40-45 adenosines for maturation, which also completes the stop codon at $3^{\prime}$ end of mRNA in most cases [26]. Bilaterian mt-mRNAs are either essentially devoid of the untranslated regions (UTRs) or tend to have very short UTRs consisting of 1-2 nucleotides flanking the mature mRNAs; a few notable exceptions such as COI, COII, ND5 and ND6 genes, which possess slightly longer $3^{\prime}$ UTRs complementary to the genes on opposite strand [23, 27]. This transcription model is, however, based on the study of a small number of bilaterians [19-22]. Recent studies on medusozoan members possessing linear mitogenomes do provide some insights into the mt-transcription in non-bilaterians $[28,29]$. However, a detailed exploration of mitochondrial RNA processing and characterization of UTRs is still lacking for most non-bilaterian animals, including anthozoans with circular mitogenomes more similar to the stereotypical animal mitochondrial genomes.
Among non-bilaterian metazoans, octocorals (Octocorallia: Anthozoa) are unique due to their atypical mitochondrial genomes. As many as five different gene arrangements have been reported among the octocorals studied so far [30-33], all with an exceptionally reduced complement of transfer RNAs (i.e. a single tRNA ${ }^{\mathrm{Met}}$ gene) and the presence of an additional gene, a mismatch repair gene $(m t M u t S)$ [13, 34], closely related to the non-eukaryotic MutS7 lineage from epsilon-proteobacteria or DNA viruses [35]. This gene has been predicted to have a self-contained mismatch DNA repair function [35], and it has been speculated to play a role in the slow rate of mtDNA evolution observed among octocorals [36, 37], and to be responsible for various genome rearrangements through intramolecular recombination [31]. Considering the evolutionary trend towards a reduced mitogenome in Metazoa [38], the occurrence of such a large gene, such as the mtMutS, occupying nearly $16 \%$ of the octocoral mitogenome is somewhat surprising. The presence of a $m t M u t S$ mRNA transcript suggested its availability for translation [35]. However, 20 years after its discovery [13] and despite of being extensively used for phylogenetic studies of octocorals [39], a thorough understanding of its transcriptional processing and maturation, and in general of its biology is lacking.

Octocoral mitogenomes exhibit five different gene arrangements, all containing a single gene for tRNA ${ }^{\text {Met }}$. Cleavage of this tRNA from the precursor polycistronic RNA would only result in linearization of precursors. The way in which the individual mitochondrial gene mRNAs are released for maturation from the long polycistronic precursor remains to be determined. In absence of knowledge on the precise boundaries of the mitochondrial mRNA in octocorals, despite all the novelties they confer, the understanding of the biology and evolution of animal mitochondria remain incomplete. Here we characterize the mitogenome transcription of an early branching non-bilaterian metazoan, the octocoral Sinularia cf. cruciata. (Alcyoniidae: Octocorallia). We describe the $5^{\prime}$ and $3^{\prime}$ boundaries and UTRs of mature mitochondrial mRNAs and characterize the transcription of the $m t M u t S$ gene. Our results provide the first glimpse to the unique features and complexity of the mitochondrial transcriptome in non-bilaterians.

\section{Results}

\section{The mitogenome of Sinularia cf. cruciata}

The complete mitogenome of Sinularia cf. cruciata was $18,730 \mathrm{bp}$ in length and included, similar to other octocorals, 14 protein-coding genes (PCGs) (ATP6, ATP8, COI-III, CytB, ND1-6, ND4L and mtMutS), two ribosomal RNAs (12S and 16S) and a single transfer RNA 


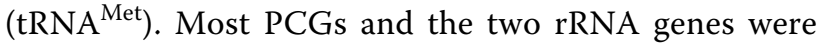
encoded on the H-strand. ATP6, ATP8, COII, COIII and tRNA $^{\text {Met }}$ were encoded on the L-strand. Gene order was consistent with that of other octocorals with mitogenome arrangement ' $A$ ' [31]. Base composition of the mitogenome was A, 30.2\%; C, 16.5\%; G, 19.3\%; T, 33.9\% and $\mathrm{G}+\mathrm{C}, 35.8 \%$. Among the 14 PCGs, the mtMutS (2982 bp) was the longest and ATP8 (216 bp) the shortest. All PCGs had ATG as the start codon, while the stop codons TAA and TAG were predominant among PCGs; $C O I$ was an exception, having an incomplete termination codon (T) (see Additional file 1: Table S1). Except for ND2 and ND4 (13 bp overlap), the remaining genes were separated by intergenic regions (IGRs) of different lengths (see Additional file 1: Table S1, Figure S1A). The longest IGR (112 bp) was found between COII and $\mathrm{COI}$, while the shortest (4 bp) was located between $12 \mathrm{~S}$ and ND1. The mitogenome of Sinularia cf. cruciata was $12 \mathrm{bp}$ shorter than that of Sinularia peculiaris and the two species had same base composition and GC content. Sequence variability within the two Sinularia species was $2.31 \%$, excluding nucleotide ambiguities $(0.05 \%)$ and gaps $(0.16 \%)$. The most variable genes were mtMutS, ND2, ND5, ND4 and COII (Additional file 1: Figure S1B).

\section{Recovering mitogenomic transcripts from RNA-Seq data} of S. cf. cruciata and other octocorals

In order to understand mtDNA expression and processing in octocorals, an RNA-Seq library of Sinularia cf. cruciata was screened for mitochondria-mapping reads (hereafter mt-reads). A total of 4153 reads out of $26 \mathrm{M}$ pairs were mapped to the sequenced $S$. cf. $\mathrm{cru}$ ciata mitogenome. This resulted in a partial mitogenome assembly covering $62.8 \%$ of its length and leaving $37.2 \%$ of the genome uncovered. With the exception of $12 \mathrm{~S}$ and $16 S$ rRNAs, which exhibited very high coverage, none of the PCGs present in the mitogenome were completely covered (Fig. 1). Despite low coverage, reads spanning IGRs were detected pointing towards the collinear expression of $C y t B-N D 6, N D 4 L-m t M u t S, N D 5-N D 4$, ATP8-ATP6, and COII-ATP8.

Additionally, RNA-Seq data from two published octocoral transcriptome studies were screened for mitochondrial reads. These included Gorgonia ventalina (Genome arrangement A; SRR935078-87) and Corallium rubrum (Genome arrangement C; SRX675792) [31, 40-42]. Hence, they provided us with the opportunity to explore mt-transcription in octocorals with different mitogenome arrangements. In case of G. ventalina, 55,783 out of $302 \mathrm{M}$ reads were mapped to the Pseudopterogorgia

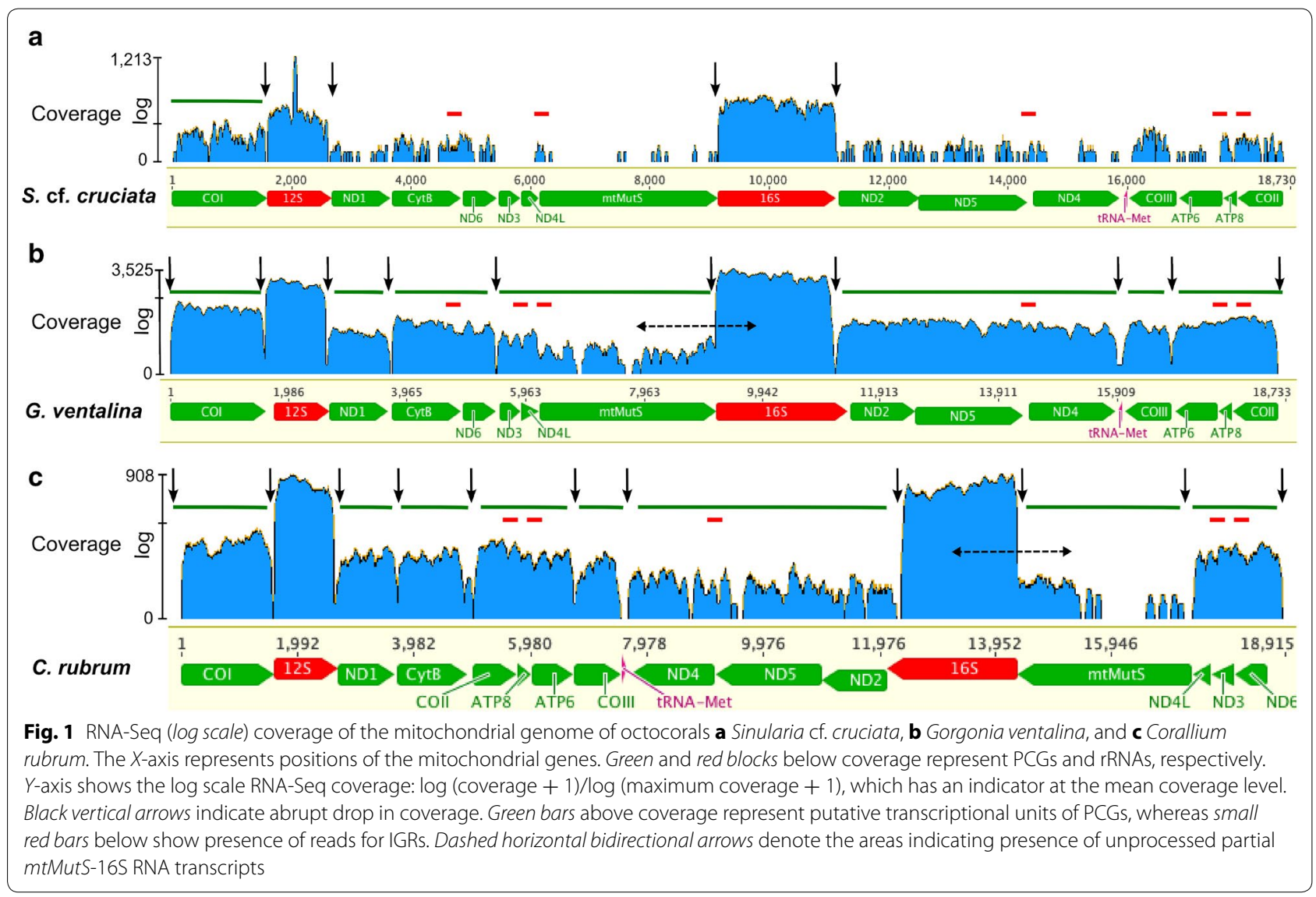


bipinnata mitogenome (NC_008157), a species closely related to G. ventalina. Almost the entire mitogenome was covered except for $2.1 \%$ uncovered sequence data, which mainly included IGRs between putative transcription units and a region of the $m t M u t S$ gene. Based on the observation of read-pairs spanning IGRs, the entire L-strand genes, namely COII, ATP6, ATP8, COIII, were detected as a collinear unit, whereas, for the $\mathrm{H}$ - strand the COI-12S-ND1, CytB-ND6, ND3-ND4-mtMutS, ND2-ND5-ND4 were detected as collinear transcriptional units. However, as judged by sudden drop in coverage at IGRs between genes and the difference in expression levels of $C O I$ and $12 S$ as well as $12 S$ and $N D 1$ it is likely that these genes are actually monocistronic units and that the detected collinearity results from sequencing of low abundance premature RNA or unprocessed intermediates of these genes.

In the case of $C$. rubrum, 17,126 out of $241 \mathrm{M}$ reads were mapped to its mitogenome (AB700136). 8.8\% (1661 nt) data was missing. $\mathrm{COI}$ was present as a single transcriptional unit whereas other PCGs were observed to occur as a collinear unit as follows: ND1-CytB, COII-ATP8ATP6-COIII, ND6-ND3-ND4L, and ND2-ND5-ND4 (with low coverage). We were unable to assign $m t M u t S$ to any of the transcription units due to small number of reads mapped to it. (see Fig. 2 for a proposed scheme of mitogenome expression for arrangement ' $\mathrm{A}$ ' and ' $\mathrm{C}$ ').

Independently of the sequencing depth, in all three analyzed transcriptomes $12 S$ and $16 S$ were the most abundant transcripts (Additional file 2). Moreover, we observed that none of the three analyzed transcriptomes contained reads to cover the complete $m t M u t S$ gene transcripts. No RNA-Seq reads could be mapped to the IGR region between COII-COI and ND6-COI in S. cf. cruciata/G. ventalina, and C. rubrum, respectively. These regions fold into a stable stem-loop structure with a $33 \mathrm{bp}$ conserved motif in all octocorals studied here (Additional file 3), and represent an inversion of polarities, thus they could function as control regions (CR)/ origin of replication (OriH) in octocorals with 'A' and ' $\mathrm{C}$ ' type mitogenomes, respectively.

\section{RT-PCR corroborates the presence of mature polycistronic} mRNA transcripts in the mitochondrial transcriptome of $S$. cf. cruciata

Depending on the sequencing depth of the RNA-Seq libraries, the RNA-Seq data may contain immature/ unprocessed precursor RNA that could lead to the detection of false polycistronic mRNAs. In order to verify the presence of genuine mature polycistronic mRNA transcripts, we conducted RT-PCR experiment using primers binding substantially up/downstream from the start/ stop codons of putative consecutive genes and amplifying their IGRs. Using DNA as a template, amplification was observed using all primer pairs screened (Table 1). Using cDNA synthesized with an anchored oligo(dT) primer as a PCR template, bicistronic and tricistronic transcripts corresponding to the CytB-ND6, ND2-ND5, and ND3$N D 4 L-m t M u t S$, COII-ATP8-ATP6, respectively, were detected corroborating the result obtained from the analysis of RNA-Seq data suggesting that these regions are polycistronic transcription units.

\section{UTR mapping of the mitochondrial protein coding genes} in S. cf. cruciata

The UTRs of several mitochondrial mature transcriptional units were mapped using $5^{\prime} / 3^{\prime}$ RACE and circularized RTPCR. The COI mRNA has a 4 bp $5^{\prime}$ UTR upstream to the start codon. The $3^{\prime}$ end of this gene, which lacks proper stop codon could not be deduced with enough certainty, but is likely that the partial stop codon is completed by polyadenylation yielding a monocistronic unit, in agreement with the transcriptome and RT-PCR results, which suggested a monocistronic nature of $C O I$.

For $C y t B$ three different $5^{\prime}$ ends were detected. One of these $5^{\prime}$ ends initiated exactly two codons ( $6 \mathrm{bp}$ ) downstream (position 3683) from the annotated start, without any $5^{\prime}$ UTR. The other two were downstream from this $5^{\prime}$ end at positions 3926 and 3970 . The first two messengers were detected using both RACE and cRT-PCR, whereas the third one was observed only with cRT-PCR. No $3^{\prime}$ end could be detected for $C y t B$ mRNA further supporting its co-expression with $N D 6$ in a bicistronic unit. Moreover, a polyadenylated ND6 mRNA $3^{\prime}$ end was detected with an 8 bp $3^{\prime}$ UTR.

Solely based on cRT-PCR, the mature mRNA ends were detected for the ND2-ND5-ND4 tricistronic unit. The $5^{\prime}$ consists of a single base before the start codon at position 11,173 (not 11,146 as it is annotated in GenBank). A $44 \mathrm{bp}$ long $3^{\prime}$ UTR was found after the stop codon at position 15,868 .

The analysis of transcriptomic data indicated the presence of a COII-ATP8-ATP6 tricistronic mRNA. RT-PCR results and end mapping corroborated this observation. COII mRNA was found to be flanked by a 3 bp $5^{\prime}$ UTR, whereas an 83 bp long $3^{\prime}$ UTR was detected after the stop codon of ATP6. The precise ends of the protein coding genes ND1 and COIII, which based on transcriptome and RT-PCR results likely are monocistronic messages, could not be determined. For more details on the UTRs of the mature mt-mRNAs of $S$. cf. cruciata see Fig. 3.

\section{The $m t$ MutS is alternatively transcribed and its transcripts are differentially expressed}

For the mtMutS, 5'-RACE mapped a 31 bp $5^{\prime}$ UTR upstream of the ND3 start codon further corroborating 


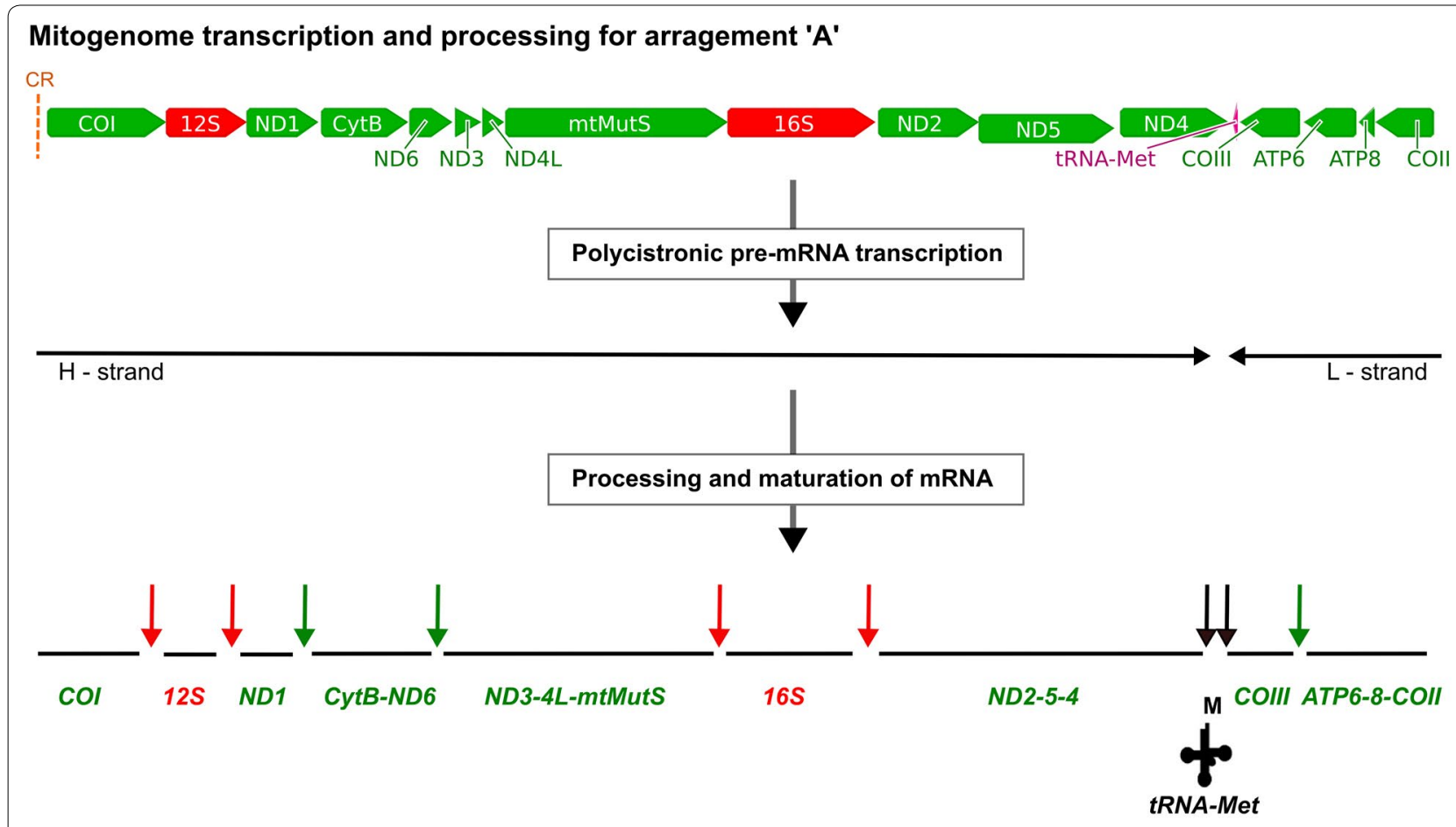

Mitogenome transcription and processing for arragement ' $C$ '

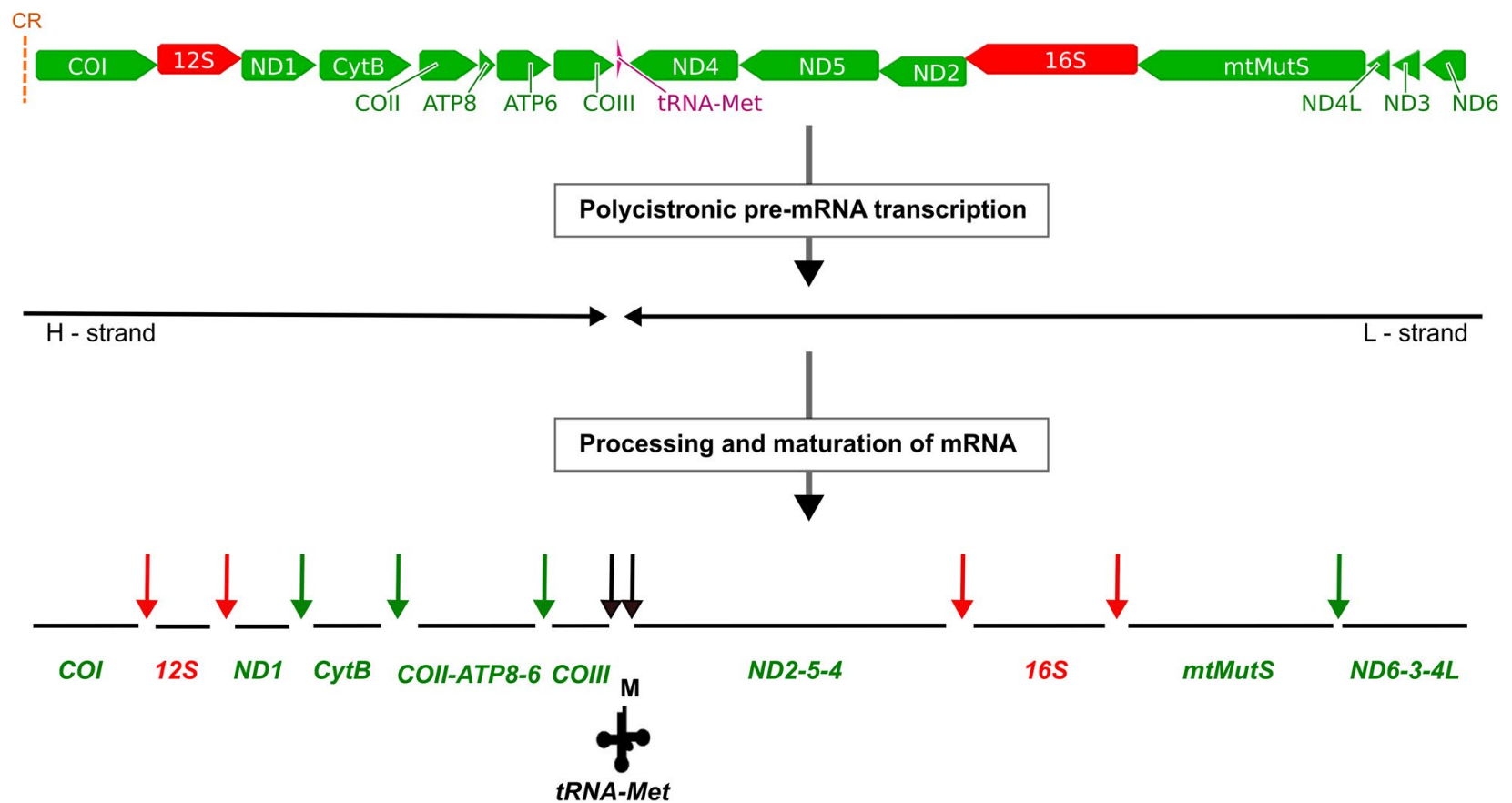

Fig. 2 Predicted model of mt-mRNA processing for mitogenome arrangements ' $A$ ' and ' $C$ ' in octocorals. The vertical dotted orange lines are the control regions (CR). The black horizontal arrows are two polycistronic pre-mRNA transcripts each. Black vertical arrows indicate excision of tRNA as per "tRNA punctuation model". Red and green vertical arrows represent additional excision sites and the resultant mature mitochondrial mRNA transcripts predicted based on our data 
Table 1 RT-PCR screening for mature mRNA transcripts spanning two or more genes

\begin{tabular}{|c|c|c|c|c|c|c|}
\hline \multirow{2}{*}{$\begin{array}{l}\text { Sr. } \\
\text { no. }\end{array}$} & \multirow{2}{*}{$\begin{array}{l}\text { Primer pair } \\
\text { forward/reverse }\end{array}$} & \multirow{2}{*}{ Regions } & \multirow{2}{*}{$\begin{array}{l}\text { Length } \\
\text { (bp) }\end{array}$} & \multirow{2}{*}{ igr+Gene ${ }^{a}$} & \multicolumn{2}{|c|}{ RT-PCR } \\
\hline & & & & & DNA & cDNA \\
\hline 1 & C2-18519/C1-839 & COII-COI & 1051 & 951 & $\square$ & $\mathbf{x}$ \\
\hline 2 & C1-743/12S-2240 & COI-12S & 1498 & 658 & $\nabla$ & $\mathbf{x}$ \\
\hline 3 & N1-3534/CB-4128 & ND1-CytB & 595 & 479 & $\nabla$ & 田 \\
\hline 4 & CB-4539/N6-4995 & CytB-ND6 & 447 & 142 & $\nabla$ & $\nabla$ \\
\hline 5 & CB-4539/N3-5625 & CytB-ND6-ND3 & 1064 & ND6+171 & $\nabla$ & 冈 \\
\hline 6 & N6-4974/N3-5625 & ND6-ND3 & 641 & 171 & $\nabla$ & $\nabla$ \\
\hline 7 & N6-4974/N3-5820 & ND6-ND3 & 859 & 389 & $\nabla$ & 冈 \\
\hline 8 & N6-4974/N4L-6120 & ND6-ND3-ND4L & 1159 & ND3+291 & $\nabla$ & $\mathbf{x}$ \\
\hline 9 & N3-5499/M-6780 & $\begin{array}{l}\text { ND3-ND4L- } \\
\text { mtMutS }\end{array}$ & 1282 & ND4L+638 & $\nabla$ & $\nabla$ \\
\hline 10 & N3-5499/M-7199 & $\begin{array}{l}\text { ND3-ND4L- } \\
\text { mtMutS }\end{array}$ & 1701 & ND4L+1057 & $\nabla$ & $\nabla$ \\
\hline 11 & N3-5499/M-8286 & $\begin{array}{l}\text { ND3-ND4L- } \\
\text { mtMutS }\end{array}$ & 2788 & $N D 4 L+2456$ & $\nabla$ & $\nabla$ \\
\hline 12 & N3-5499/M-8826 & $\begin{array}{l}\text { ND3-ND4L- } \\
\text { mtMutS }\end{array}$ & 3328 & ND4L+2684 & $\nabla$ & $\nabla$ \\
\hline 13 & M-8714/16S-9396 & mtMutS-16S & 683 & 259 & $\nabla$ & $\nabla$ \\
\hline 14 & $16 \mathrm{~S}-10954 / \mathrm{N} 2-11753$ & $16 S-N D 2$ & 800 & 639 & $\nabla$ & 田 \\
\hline 15 & N2-12177/N5-12612 & ND2-ND5 & 448 & 106 & $\nabla$ & $\nabla$ \\
\hline 16 & N5-12961/N4-15314 & ND5-ND4 & 2354 & 990 & $\nabla$ & $\nabla$ \\
\hline 17 & N4-14807/C3-16468 & ND4-COIII & 1662 & 598 & $\nabla$ & $\mathbf{x}$ \\
\hline 18 & N4-15714/A6-17048 & ND4-COIII-ATP6 & 1335 & COIII+225 & $\nabla$ & 冈 \\
\hline
\end{tabular}

$\square$, positive amplification; $\mathbf{x}$, no amplification

Primer codes $=$ first part indicates gene and number indicates $5^{\prime}$ end of primer corresponding to $S$. cf. cruciata mitogenome

a Indicates bps into the gene downstream to the igr 
a

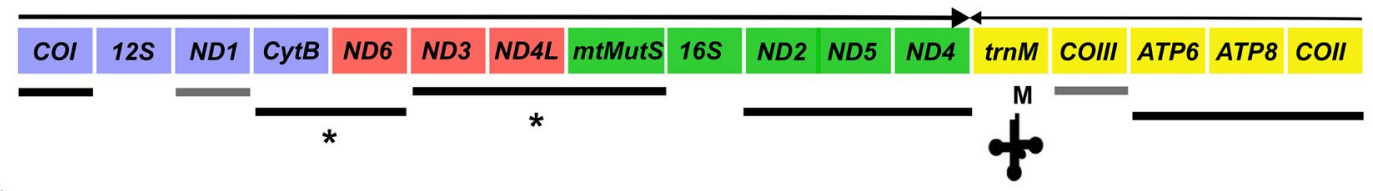

b

COI

mIDNA: AGAGTAAGCATGAACAAA

mRNA: $\quad$ AAGCAUGAACAAA

$\#$ coll

mtDNA: TAGAGAAGAATGAAAACT

mRNA: $\quad$ AGAAUGAAAACU

\# $\mathrm{M}$ K T

CytB-ND6*

3683

mtDNA: CGCATGCATATGGAATCA

mRNA: $\quad$ CAUAUGGAAUCA

$\#$ M E S

ND2-ND5-ND4

11173

mIDNA: ATGTGGGTGCATAGCCCCTGGCATACTATGGAATTA mRNA:

UAUGCAAUUA

M E L

C

\section{CytB-ND6}

mtDNA: CACCTTTAGGTGCCTTCTGG

mRNA: CACCUUUAGGUGCCUUC-Poly(A)

$\mathrm{H} \quad \mathrm{L} * \overline{5} 439$

\section{ND2-ND5-ND4}

mtDNA: TTTTTATAATTTTCCTCTTTCCTGTACCTATTTGGTTTATATGTGTATCTATTTTG

mRNA: UUUUUAUAAUUUUCCUCUUUCCUGUACCUAUUUGGUUUAUAUGUGUAUCUAUU-Poly(A)

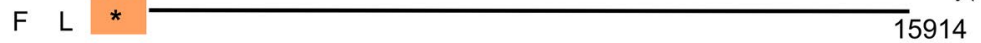

\section{COII-ATP8-ATP6}

mtDNA: CTACATTAACCCATACTTAGAATAATTGTTGGGTAGGAGTATTAGTCTCCGCTTGATTCCCCGCCGGGGAGCCA mRNA: CUACAUUAACCCAUACUUAGAAUAAUUGUUGGGUAGGAGUAUUAAUCUCCGCUUGAUUCCCCGCCGGGGAG-Poly(A)

d

\section{3}

3926

3970

Fig. 3 Mapped $5^{\prime}$ and $3^{\prime}$ ends of mature mitochondrial mRNAs. a Schematic representation of Sinularia mito-transcriptome arrangement. Arrows above show transcription orientation. Lines below denote the transcription units (mono- and polycistronic transcripts). Black lines below indicate transcription units for which one or both the ends are known, whereas grey lines (below ND1 and COIII) indicate units for which ends remained unknown. Asterisk shows the transcription units for which alternate ends were detected. b Summary of 5' end mapping for mt-mtRNAs. The 5' UTR regions are underlined. Shaded boxes depict start codons. Hash indicates detection using both, RACE and cRT-PCR methods. Nucleotide positions of the first base of the start codons are indicated. c Summary of $3^{\prime}$ end mapping for mt-mtRNAs. The $3^{\prime}$ UTR regions are underlined. Colored boxes highlight stop codons. Nucleotide positions of the last base of stop codons are indicated. d Alternative starting positions (5' ends) of CytB-ND6 mRNA 
that this gene is transcribed as a tricistronic unit together with ND3 and ND4L (Fig. 4a, b). Using several mtMutSspecific primers we were unable to detect any alternate $5^{\prime}$ end using RACE PCR. However, using circularized RT-PCR an internal $m t M u t S 5^{\prime}$ end was detected $387 \mathrm{bp}$ downstream (position 6542) of the annotated mtMutS start codon. The $3^{\prime}$ end for this particular transcript was at a position 8950 , which is $188 \mathrm{bp}$ upstream to the annotated stop codon. This transcript ended with UAA and is clearly followed by a polyA-tail. However, this stop codon is not in frame.

Somewhat surprisingly, at least six different $3^{\prime}$ ends were detected for the $m t M u t S$ gene mRNA using RACE and cRT-PCR. These were found to end at position 6746, 6771, 6911, 8761, 8950 (as described above), and 8977 besides the annotated in-frame stop codon at position-9135 (Fig. 4c). These messages are polyadenylated and end with GAA or UAA. Notably, none of these endcodons are in-frame. RT-PCR screening confirmed the presence of all possible transcript variants described above (see Additional file 4: Table S2 for primer details).
Further confirmation of alternative polyadenylation (APA) of $m t M u t S$ messengers in the normal mt-mRNA pool was attained using RHAPA (RNase $\mathrm{H}$ alternative polyadenylation assay).

The $m t M u t S$ transcripts were differentially expressed. The central region, which includes a partial domain III of $m t M u t S$, was $6.35 \pm 0.3$-fold more abundant than the $3^{\prime}$ end of the gene whereas the expression of the transcript containing the $5^{\prime}$ part of the $m t M u t S$ mRNA was $1.8 \pm 0.15$-fold higher than that of the extreme $3^{\prime}$ end of the transcript (Fig. 5). This observation suggests the existence of different variants of the same gene under normal conditions in the mature mt-mRNA pool of Sinularia cf. cruciata (For details on the primers used for this assay see Additional file 4: Table S2 and Additional file 5 for results).

\section{Antisense ATP6 mRNA}

Using $5^{\prime}$ RACE we detected antisense mRNA transcripts complementary to the ATP6. Five different starting points were determined for these transcripts (i.e. positions

a

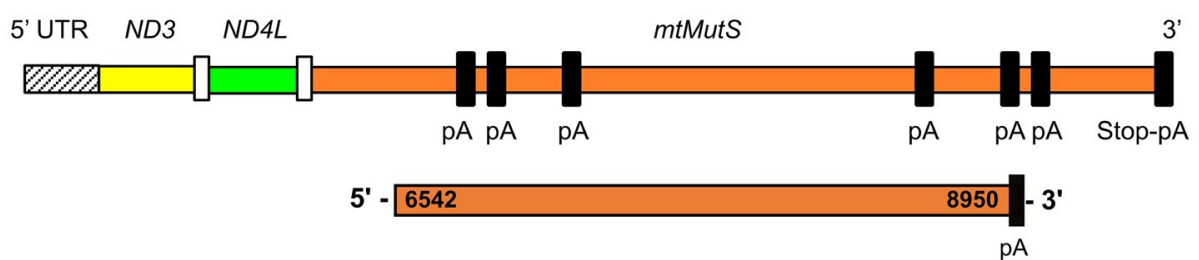

b

ND3-ND4L-mtMutS*

mtDNA: AAGTGTTAATGCACGTCTCCGCTTAAGAAACATGGAGTTC

mRNA: AAGUGUUAAUGCACGUCUCCGCUUAAGAAACAUGGAGUUC

\#

M E F

C
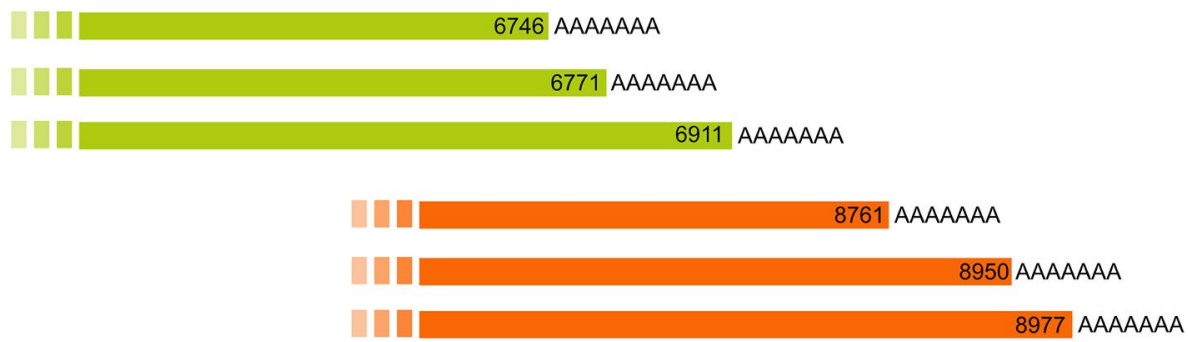

Fig. 4 The mtMutS mRNA transcripts. a Schematic of the mtMutS gene as a tricistronic transcription unit with different poly(A) tail positions (not to scale) shown as dark blocks. pA = poly(A)-tail. Below is the internal mtMutS transcript. b The $5^{\prime}$ end of ND3-ND4L-mtMutS tricistronic transcript. The $5^{\prime}$ UTR region is underlined. Shaded box indicate the start codon. The arrow above indicates 11 bp deletion in S. cf. cruciata compared to S. piculiaris. Hash indicates detection using both, RACE and CRT-PCR methods. c Alternatively polyadenylated mtMutS mRNAs; the position of the poly(A) start is indicated 


\section{S. cf. cruciata "Sinularia sp.}

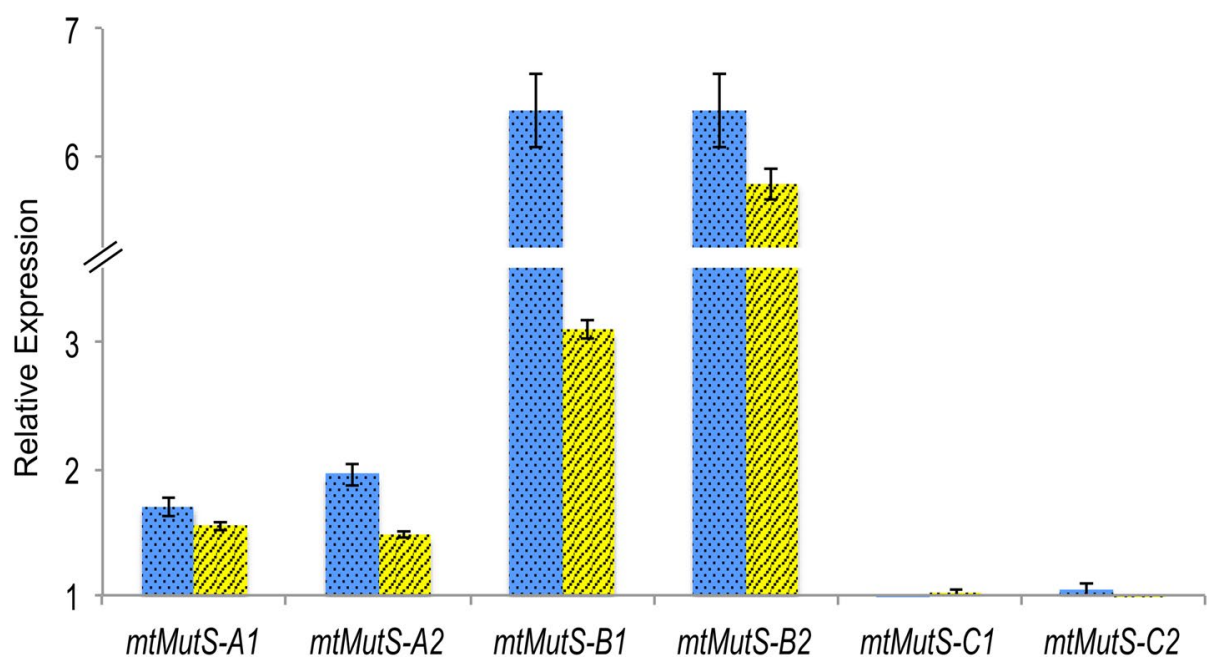

Fig. 5 Relative quantification of alternate $m t M u t S$ mRNA transcript abundance. Quantification of alternatively polyadenylated $m t M u t S$ transcripts. The ACTB gene served as reference. The comparison was performed against the $3^{\prime}$ region cleaved-off after RNase H digestion ( $m$ tMutS-C1, C2) (see Additional file 4: Table S2 for primer details). Data shows relative expression \pm SD of technical triplicates for two Sinularia species

$16,837,16,866,16,870,16,891$, and 16,920), which were longer than $200 \mathrm{bp}$ (reverse primer used binds the antisense messenger at position 17,048 ), polyadenylated (i.e. reverse transcribed using anchored oligo-dT), and lacked open reading frames (ORFs). Therefore, this RNA species can be categorized as long noncoding RNA (lncRNA) [43]. Antisense strand-specific RT-PCR of an internal region of these $\ln A$ ATP 6 transcripts further corroborated their presence (see Additional file 6).

\section{Discussion}

Using different experimental approaches we describe the mitogenome expression patterns of an early branching animal. The precise ends of most mature mt-mRNAs were assessed for the first time in octocorals. Most mature protein-coding mRNAs detected were bicistronic or tricistronic units, with the exception of COI, ND1 and COIII. The occurrence of polycistronic mature mRNAs potentially stems from the paucity of tRNA punctuation marks in the octocoral mitogenome. The majority of mature transcription units were found to possess $5^{\prime}$ and $3^{\prime}$ UTRs, contrary to what is known for bilaterians in general. Moreover, the occurrence of APA sites of the $m t M u t S$ mRNA transcript and long non-coding antisense ATP6 RNA (lncATP6) provide a glimpse into a unique and potentially complex mitochondrial transcription mechanism in octocorals and likely in other early branching metazoans with non-canonical mitogenomes.

The evolution of specific mechanisms for the expression of the mitogenome was necessitated by the reduced gene repertoire and compact nature of this crucial cell organelle with its own genome evolved from an $\alpha$-proteobacteriumlike ancestor $[44,45]$. In cnidarians, which contrary to a typical animal mitogenome containing 37 (13 PCGs, 2 rRNA and 22 tRNA) genes in total, harbor only $16 \pm 2$ (13 or 14 PCGs, two rRNAs and one or two tRNA) genes, reduction in gene content, but not genome size, is remarkable to some extent. Our understanding of the mitogenome transcription/expression patterns and regulation is currently limited to a handful of bilaterian members of the animal kingdom [19-21]. However, such attempts are lacking for non-bilaterians. Information on the mitochondrial transcriptomes of sea anemone [46], hydrozoan [28] and jellyfish [29] have shown the potential complexity of mitochondrial transcription and highlighted the need for a better understanding of the evolutionary processes leading to different strategies of mitochondrial transcription and regulation.

The processing of the mt-tRNAs interspersed in the mitogenomes of most animals provide a mechanism to liberate monocistronic protein-coding mRNAs from polycistronic precursors, leading to their maturation and availability for translation [24]. Most studies in animals so far point to the generality of the tRNA punctuation model of mitochondrial mRNA processing with only the occurrence of two bicistronic transcription units (i.e. ATP8-ATP6 and ND4L-ND4) apparently due to the overlap of their ORFs [20,21,23]. Cnidarians, however, possess only one or two tRNA genes in their mitogenomes. Our observation of four polycistronic units 
comprising 11 (out of 14) different PCGs is staggering, and provides evidence for a potentially unique mechanism of mitochondrial mRNA processing, expression and regulation in octocorals. These findings may also apply to other animals exhibiting a paucity of mt-tRNA genes in their mitogenome, for example, chaetognaths [47], some demosponges [48] and other cnidarians [49].

The use of RNA-Seq data provides a unique opportunity to explore mitogenome expression in non-model organisms such as non-bilaterian metazoans. Nevertheless, we observed that very few reads mapped to the published/sequenced mitochondrial genomes $(<0.018 \%)$. This has been observed before using different NGS methods in sea anemones (0.053\% reads) [46], and hydrozoans $(<0.003 \%$ reads) [28], where very few reads could be identified as mitochondrial. We assembled almost the entire mitogenome of $G$. ventalina with the exception of $<3 \%$ of its nucleotides mostly belonging to IGRs flanking mature transcription units and to the $m t M u t S$ gene. In the case of Corallium and Sinularia, and despite the difference in the number of reads available for mapping in these two species, mapping did not result in a sufficient number of reads to produce reliable contigs. Yet, the presence of read-pairs spanning multiple genes allowed us to postulate the presence of polycistronic mt-mRNAs in these species. Emblem et al. [46] suggested that low mitogenome copy number per haploid nuclear genome results in low-level expression of mitochondrial mRNAs and is responsible for the depleted number of mitochondrial reads observed in three different sea anemones. This may explain our results as well. The poor coverage observed for the $m t M u t S$ gene remains puzzling. The absence of a full $m t M u t S$ transcript can be attributed to low coverage in case of $S$. cf. cruciata. However, a full $m t M u t S$ is also absent from the other transcriptomes analyzed that have higher sequencing depths. It should also be noted that there are marked differences in the mtMutS coverage despite of similar sequencing depths in these two transcriptomes (relative well-covered with higher expression in G. ventalina but not in C. rubrum) (see Fig. 2). Large size, the occurrence of APA, and lack of a persistent requirement of the gene product under normal physiological conditions (relative to the genes involved in oxidative phosphorylation) potentially explain the low expression of the $m t M u t S$ gene under such conditions.

Previous studies on octocorals have proposed the COII-COI IGR as potential CR/oriH in the octocorals with mitogenome arrangement ' $A$ ' [30-32]. The observed absence of RNA-Seq reads in G. ventalina supports this proposition. The presence of very similar stem-loop structure in the ND6-COI IGR in C. rubrum, along with absence of RNA-Seq reads in this region of this species, suggests that this IGR is the potential CR in octocorals with ' $\mathrm{C}$ ' type mitogenome arrangement and corroborates earlier predictions in this regard [32, 42] (Additional file 3).

Untranslated regions (UTRs) flanking the mature mRNA transcripts play a crucial role in the post-transcriptional regulation of gene expression [50]. In mitochondria, mature mRNA transcripts are generally devoid of UTRs, or these are only few $(\leq 3)$ nucleotides long [23]. The presence of $5^{\prime}$ UTRs for transcriptional units such as COI, ND3-ND4L-mtMutS, ND2-ND5-ND4, and COIIATP8-ATP6 mRNA, and of $3^{\prime}$ UTRs for CytB-ND6, ND4, and COII-ATP8-ATP6 suggests a putative role of these elements in the regulation of these genes and represent, to our knowledge, the first report of the presence of long UTRs in the mature mt-mRNAs of non-bilaterians.

Different studies have detected at least five mitogenome arrangements in octocorals so far, all of which appear to preserve four conserved gene blocks. The inversion or translocation of one of these blocks at a time is proposed to have led to five different mitogenome arrangements $[31,33]$. It has been suggested that the occurrence of the genes in conserved clusters is selectively advantageous, for instance, as the genes can be co-transcribed and processed in a similar way $[31,51]$. However, the evidence on selection favoring a particular mitochondria gene order is sparse in cnidarians, as they exhibit high diversity of mitogenome arrangements with no sharing of gene boundaries, particularly in the subclass Hexacorallia [31]. The transcriptional units we detected encompass genes from two distinct adjacent gene blocks (e.g. the polycistronic transcription units $C y t B-N D 6$ and ND3$N D 4 L-m t M u t S)$, contradicting the hypothesis of cotranscription as a selective force in keeping these genes together in conserved blocks in the mitochondria of octocorals. Evidence for selection favoring a particular mitochondrial gene order in cnidarians and other metazoan taxa is scarce as well $[31,52]$. Hence, maintenance of synteny within the four conserved gene blocks detected so far appears to result from the lack of recombination-hotspots that promote genome rearrangements within, and/ or their prevalence between these conserved gene blocks. Expression studies in future would be required to corroborate this initial observation. However, our results indicate that different mitogenome rearrangements detected in octocorals have different mature mt-mRNA transcript structures and transcriptional patterns (see Corallium vs. Sinularia-Gorgonia in Fig. 2). This transcriptional diversity highlights the potential complexity of mitochondrial transcription among non-bilaterians.

Assuming the tRNA punctuation model holds true, in three out of five mitogenome arrangements observed in octocorals (i.e. A, C and D), the tRNA ${ }^{\text {Met }}$ lies at the end of either $\mathrm{H}$ - or L-strand and its processing would liberate 
both coding and non-coding parts of the polycistronic precursor RNA. In S. cf. cruciata, the simultaneous or sequential processing of rRNAs could provide a mechanism for the liberation of COI and ND2-ND5-ND4. However, the excision of other transcription units from the polycistronic precursor remains to be explained. Secondary structures such as stem-loops are likely involved in maturation of pre-mRNA in octocorals, as is the case in hydrozoans and other animals $[19,28]$. In S. cf. cruciata, IGRs where excision is required in order to liberate detected transcriptional units (i.e. $N D 1-C y t B$, ND6-ND3, COIII-ATP6) form one or more stem-loop structures (Additional file 7A). We pose that the enzymes involved in mRNA maturation recognize the conserved 11 bp motifs (Additional file 7B) present in the IGRs flanking transcription units and cleave them from the precursor to be available for maturation. This motif is absent in the IGRs between individual genes within the above-mentioned transcription units.

The mtMutS gene present in octocorals is thought to underpin several peculiar processes not present in other animal mitochondria. The presence of a large gene not involved in energy production within a streamlined organelle genome dedicated to this task is mysterious as well as interesting. The second largest gene in Sinularia cf. cruciata mitogenome, ND5 (1818 bp long) is known to be the most tightly regulated protein-coding gene in other animals [53]. Thus we hypothesize that the transcription of $m t M u t S$ is tightly regulated as well. In favor of this hypothesis, we observed distinct $m t M u t S$ variants resulting from the use of different internal polyadenylation sites within the $m t M u t S$ gene. In contrast to its function in plant mitochondria and bacteria, which polyadenylate RNA to promote their degradation [54], polyadenylation is used in mammals to provide stability to the mature mRNA and create the stop codon, if it is not complete [26]. Polyadenylated truncated transcripts destined to degradation have also been detected in mammals [27], but, their abundance is low and they are generally difficult to detect using standard methods. All the $m t M u t S$ variants reported here were readily detectable indicating their potential functional role.

Interestingly, the alternate $m t M u t S$ transcripts were differentially expressed with a transcript variant encompassing Domain III and V of $m t M u t S$ (position 6542-8950) being more abundant under normal conditions than either the $5^{\prime}$ or $3^{\prime}$ end regions of the gene. These domains are either structurally important (e.g. Domain III) or have important biochemical functionality (e.g. ATPase; Domain V) $[35,55]$. APA plays a crucial role in regulating gene expression [56]. Hence, APA of the mtMuts gene may have a regulatory function allowing a tight control of the expression of this gene in octocoral mitochondria.
The use of the RHAPA method avoided a limitation of Northern blotting that has a tendency to detect unprocessed transcripts and/or degradation intermediates. Specifically, transcripts having partial $m t M u t S$ continued into the $16 \mathrm{~S}$ were readily detected with cRT-PCR, and also visible in the G. ventalina and C. rubrum transcriptome data (Fig. 1). In addition, RHAPA also proved helpful in determining the relative abundances of APA transcripts when coupled with qPCR. However, this resulted in lack of information on sizes of each of the alternatively polyadenylated transcripts. Attempts to obtain size information on each type of alternatively polyadenylated $m t M u t S$ transcripts using a common $5^{\prime}$ primer and oligo(dT) led to the detection of false alternate transcripts of different sizes with apparent artificial deletions in the sequence. This appears to be caused by the presence of direct repeats in this fairly long gene, coupled with the templateswitching property of the reverse transcriptase (RT) used for cDNA synthesis, a phenomenon reported earlier by Cocquet et al. [57]. Additional efforts involving target RNA capture methods [58] would be needed to determine the sizes of individual alternate $m t M u t S$ transcripts in the future. The precise start and end points of each $m t M u t S$ mRNA variant deserve to be determined to better understand how the start-stop codons are chosen during translation. Additionally, protein studies need to be conducted in order to corroborate the localization and functionality of these transcripts and their products.

Long noncoding RNAs (lncRNAs) have been recently described in the mitochondria of mammals, primarily for $N D 5, N D 6$ and $C y t B$, and have been shown to interact with their mRNA complements stabilizing them and/or blocking the access of mitochondrial ribosomes, thereby inhibiting translation [59]. The presence of an lncRNA transcript for ATP6 (lncATP6) in Sinularia mitochondria is striking, and indicates that the regulation of mitochondrial expression using lncRNAs evolved early in Metazoa and is ancient.

More than $99 \%$ of the mitochondrial proteome is encoded by the nuclear genome [60]. The loss of mttRNAs in cnidarians is suggested to have occurred in association with the loss of nuclear-encoded mt-aminoacyl-tRNA synthetases [61] and indicates a greater nuclear dependency in cnidarians relative to other animals. The retention of a single mt-tRNA for formyl-methionine is interesting and likely reflects its very specific mitochondrial function or the necessity of an excision starting point that triggers the mt-mRNA maturation cascade. Our findings, together with the general paucity of tRNAs and the varied mitogenome rearrangements observed in octocorals indicate a highly complex and perhaps a unique system for mRNA processing in the mitochondria of these organisms. 


\section{Conclusions}

Recent studies on the human mitochondrial transcriptome revealed an unexpected complexity in expression, processing, and regulation of mt-mRNAs $[19,62]$. Our results shed first light on the potentially more complex nature of these processes in the mitochondria of early branching animals by virtue of their "special" and diverse mitogenomes. Overall, due to the lack of tRNA punctuation marks, mitochondrial mRNA processing in octocorals appears to be drastically different. The presence of polycistronic mature mRNAs for the majority of genes provides evidence for the complexity of the transcription process in these animals. The occurrence of alternately polyadenylated transcripts for the $m t M u t S$ gene and their differential expression, the existence of $5^{\prime}$ and $3^{\prime}$ UTRs, and the presence of lncATP6 transcripts are additional features highlighting the diverse set of post-transcriptional modifications and regulatory mechanisms used among octocorals. More research will contribute to better understand the mitochondrial biology of early branching animals from a functional perspective. This will certainly increase our knowledge on the innovations that shaped the evolution of these organisms.

\section{Methods}

\section{Specimens}

Coral colonies were obtained from a commercial source and maintained in a closed circuit seawater aquarium at Molecular Geo- and Palaeobiology lab, LMU, Munich. Two species of the genus Sinularia were used in this study. Sinularia cf. cruciata (Lab Voucher Code: GW1725) was utilized for the majority of the experiments, whereas additionally, a second Sinularia sp. (Lab Voucher Code: GW2911) was used for RT-PCR screening, RHAPA and antisense mRNA detection (see below). All the references to the nucleotide positions refer to the full mitochondrial genome of Sinularia cf. cruciata (GenBank Accession: KY462727), which was sequenced completely (details are provided in Additional file 1).

\section{Total RNA extraction and CDNA synthesis}

TRIzol reagent (Invitrogen, USA) was utilized for the extraction of total RNA as per the manufacture's instructions. RNA was dissolved in $100 \mu \mathrm{l}$ DEPC treated water and contaminating DNA was eliminated from RNA extracts by performing a DNase (RQ1 RNase-free DNase, Promega, USA) treatment at $37{ }^{\circ} \mathrm{C}$ for $30 \mathrm{~min}$. Treated RNA was purified after inactivation of the DNase and its purity was determined using a Nanodrop ND-1000 spectrophotometer (Thermo Fisher Scientific, USA). RNA samples with absorbance at OD260/280 and
OD260/230 ratios $\sim 2.0$ were used for further analysis. RNA integrity was also verified by $1 \%$ agarose gel electrophoresis as well as using a Bioanalyzer (Agilent Inc.). RNA extracts with a RIN value $\geq 7.5$ were used for cDNA synthesis (data not shown); these extracts were stored at $-80^{\circ} \mathrm{C}$ until use.

\section{RNA-Seq and read mapping}

RNA-Seq reads for Gorgonia ventalina (SRR935078SRR935087) and Corallium rubrum (SRR1552943SRR1552945 and SRR1553369) were downloaded from NCBI's Short Read Archive, imported in Geneious and mapped against the mitochondrial genomes of Pseudopterogorgia bipinnata (NC_008157), in the case of G. ventalina, or Corallium rubrum (AB700136). In the case of Sinularia cf. cruciata ca. $26 \times 10^{6} 50$ bp pairs of reads were sequenced, imported in Geneious ${ }^{\circledR}$ 8.1.8 (Biomatters) [63] and mapped to the mitochondrial genome we sequenced for this species. The mapping was done using a low sensitivity strategy that avoids remapping reads to previously build contigs based on the previous mapping rounds. The mapping results were screened in Geneious to find gaps in coverage flanking putative transcription units. Additionally, we screened the mapped reads to assess whether read pairs spanning adjacent genes could be found among the sequences. These reads, i.e. read-pairs linking adjacent genes and spanning intergenic regions, were taken as evidence of collinearity.

\section{Reverse-transcription PCR (RT-PCR)}

RNA extracts were PCR controlled in order to detect amplifiable levels of small DNA fragments. Only RNA extracts devoid of any amplification were used in RTPCR experiments. For each sample, $\sim 1 \mu \mathrm{g}$ of total RNA was reverse transcribed in $20 \mu \mathrm{l}$ reactions using the ProtoScript $^{\circledR}$ II First Strand cDNA Synthesis Kit (New England Biolabs, USA) with an anchored oligo-(dT) primer and following the manufacture's instructions.

RT-PCR sequencing primers were designed using the sequenced mitochondrial genomic sequence of Sinularia cf. cruciata (GenBank Accession: KY462727). Screening for the presence of the polycistronic mRNAs was done using the primers enlisted in Table 1.

\section{Analysis of $5^{\prime}$ and $3^{\prime}$ ends}

Two different approaches were used to determine the transcript ends of mature mitochondrial mRNA species.

\section{Circularized RT-PCR (CRT-PCR)}

Isolation of mRNA from total RNA was performed using Dynabeads ${ }^{\circledR}$ mRNA magnetic beads (Invitrogen). $100 \mathrm{ng}$ of polyA-selected mRNA as well as total RNA 
were circularized using T4 RNA ligase I (New England Biolabs) following the manufacture's protocol. The circularized RNA was purified and used for cRT-PCR and $5^{\prime} / 3^{\prime}$ end screening using the method described earlier [64]. Briefly, cDNA synthesis was performed as described above using gene-specific reverse primers binding near the $5^{\prime}$ end of linear RNA resulting in production of first strand that contains $5^{\prime}$ end, the ligation site, and the $3^{\prime}$ end of the molecule. These first strands were subjected to PCR amplification using specific primer pairs (see Table 2 for primer details).

\section{Rapid amplification of CDNA ends ( $5^{\prime}$ and $3^{\prime}$ RACE)}

First strand synthesis was performed to obtain the template for $5^{\prime}$ and $3^{\prime}$ RACE PCRs using SMART ${ }^{\mathrm{TM}}$ RACE cDNA Amplification Kit (CLONETECH Inc.) following the supplier's protocol. Approximately $\sim 1 \mu \mathrm{g}$ of total RNA was used to obtain two separate pools of 5'-RACEReady cDNA and 3'-RACE-Ready cDNA. RACE PCR reactions were performed using different gene-specific primers paired with adaptors primers as per the supplier's instructions (see Table 2).

\section{Cloning, sequencing and sequence analysis of amplified products}

Amplified products were either extracted from 1\% agarose gels or purified using the NucleoSpin Gel and PCR Purification Kit (MACHEREY-NAGEL, Germany) and cloned using a TOPO TA Cloning Kit (Invitrogen, USA). The clones obtained were PCR amplified, precipitated and sequenced using ABI BigDye v3.1 sequencing chemistry on an ABI 3730 DNA Analyzer Sequencing instrument. Sequences obtained were analyzed and aligned to the mitogenomes of S. piculiaris (NC_018379) and the sequenced S. cf. cruciata (Accession No. KY462727) using Geneious ${ }^{\circledR}$ 6.1.6 software (Biomatters) [63].

\section{Detection and quantification of alternative polyadenylation (APA)}

RNase $\mathrm{H}$ alternative polyadenylation assay or RHAPA [65] was employed to determine and quantify alternative polyadenylation (APA) of the mtMutS mRNA transcripts. For the first time, we coupled this assay with quantitative real-time PCR technique (qPCR),

Table 2 Primers used in this study

\begin{tabular}{|c|c|c|c|c|}
\hline Gene & 5'RACE & Sequence & $3^{\prime}$ RACE & Sequence \\
\hline \multicolumn{5}{|c|}{ A. Primers used in $5^{\prime}$ and $3^{\prime}$ RACE amplification } \\
\hline \multirow[t]{2}{*}{$\mathrm{COI}$} & $C 1-839^{a}$ & ATCATAGCATAGACCATACC & & \\
\hline & C1-1144 & CATAGTGGAAGTGAGCTACTAC & & \\
\hline$C y+B-N D 6$ & CB- $4128^{\mathrm{a}}$ & GCTCCCCAAAAGGACATTTGTC & & \\
\hline \multirow[t]{5}{*}{ ND3-ND4-mtMutS } & N3-5602 & CACATTCATAGACCGACACTT & N4L-6071 & GCCATTATGGTTAACTATTAC \\
\hline & M-6402 & ACGAAGCAACTTGTTCAATGG & M-6363 & ATTGAACAAGTTGCYTCGTTACTTG \\
\hline & $M-6719$ & CCGGGTTACTTTGTCCCTGTCCG & $M-8714$ & GCCCTCTCAATATGGCATTG \\
\hline & & & M-6655 & CAGCCATGAATGGGCATAG \\
\hline & & & M-8067 & GCATTAAGCGGGGCTATTGCGG \\
\hline COII & C2-18519 & CCATAACAGGACTAGCAGCATC & & \\
\hline Gene & $3^{\prime}$ Facing & Sequence & $5^{\prime}$ Facing & Sequence \\
\hline \multicolumn{5}{|c|}{ B. Primers used in CRT-PCR } \\
\hline $\mathrm{COI}$ & C1-1324 & TACTCGGATTTCCCTGATGC & C1-251 & AACCAATTTCCGAATCCTCCG \\
\hline$C y t B-N D 6$ & N6-4962 & TTTGGTTAGTTATTGCCTTT & CB-4128 ${ }^{\mathrm{a}}$ & GCTCCCCAAAAGGACATTTGTC \\
\hline ND3-ND4-mtMutS & M-8624 & TGATTCGCCAGTTCGGTGCT & $M-6780^{a}$ & TTAAACCTACCCCCGAGTCC \\
\hline ND2-ND5 & N5-14119 & GCTCAGTTTGGAAGTTTGGC & N2-11753 & ACATCGGGAGCCCACATA \\
\hline ND4 & N4-15714 & TTTTGGGCAACTTTCTCC & N4-14532 & CAGAGACCACTCTAACGCTTGTTG \\
\hline COII-ATP8-ATP6 & C2-18161 & GGTTGAAGGTCACTCGTAGGTATC & A6-17048 & GGGTTCGCAATGATTAGTAATGGAATGT \\
\hline Gene & & & & Sequence \\
\hline \multicolumn{5}{|c|}{ C. Gene-specific primers used for RT for CRT-PCR analysis } \\
\hline \multicolumn{3}{|c|}{ ATP6 } & 092 & TTAGCAGCCAATCGAACACC \\
\hline \multicolumn{3}{|l|}{ ND4 } & 295 & GCGTCTACCTGTCTGCAAGT \\
\hline \multicolumn{3}{|l|}{ mtMuts } & & CATTTCGGGATGGTAGCTCC \\
\hline
\end{tabular}

a Indicates the other primers used for RT during CRT-PCR 
which allows for the accurate estimation of the abundance of the alternative transcripts. The oligonucleotide $5^{\prime}$-CATTTCGGGATGGTAGCTCC- $3^{\prime}$ was used to remove the $3^{\prime}$ end of the polyadenylated complete mtMutS messenger. This primer hybridizes to the mtMutS mRNA between positions 8807 and 8826 . After hybridization, the DNA-RNA hybrids were digested with RNase $\mathrm{H}$ and the resulting mRNA was purified using RNA Clean \& Concentrator ${ }^{\mathrm{TM}}$ kit (Zymo Research) and reverse transcribed using oligo(dT) as described above. Only alternatively polyadenylated $m t M u t S$ forms should be present in the resulting cDNA after RNase $\mathrm{H}$ digestion. A control RT-PCR using primers binding to the adjacent regions of the RNase $\mathrm{H}$ digested site ensured the successful digestion of the $3^{\prime}$ including and poly(A) tail of the mature, full $m t M u t S$ mRNA species. Afterwards, a quantitative real-time PCR (qPCR) assay was performed to determine the abundance-levels of the transcripts using primers binding upstream and downstream of digested mRNA region. For primer details see Additional file 4: Table 2.

\section{Strand-specific RT-PCR}

Strand-specific RT-PCR was performed as described previously [66] for the detection of antisense RNA transcripts of the ATP6 gene in both Sinularia species. In earlier, RACE experiment, we observed ATP6 clones containing a RACE adaptor ligated at $3^{\prime}$ end of $5^{\prime}$ RACEready cDNA. This suggested the presence of an antisense transcript for this gene, as it has also been noted previously in porcine brain [67]. Two antisense strandspecific primers were used for cDNA synthesis (AAR1. 5'-TTACTCCTACTGCCCATATTG- $3^{\prime}$ and AAR2. $5^{\prime}$-TGTAGTTCGGATAATTGGGGG-3'), whereas a sense strand-specific primer (SAF. 5'-TTAGCAGCCAATCGAACACC-3') and an anchored oligo(dT) were employed separately for first strand synthesis. For RTPCR, the primer AAR1-SAF pair was used.

\section{Quantitative Real-time RT-PCR (qPCR) and data analysis} The Rotor-Gene Q 2plex system (QIAGEN) was utilized for qPCR experiment. The KAPA SYBR FAST universal mastermix (Peqlab) was used in $15 \mu \mathrm{l}$ reactions containing $1 \mu \mathrm{l}$ diluted cDNA, $7.5 \mu \mathrm{l} 2 \mathrm{X}$ mastermix, and 250$400 \mathrm{nM}$ of each primer. A two-step qPCR including an initial denaturation step of $3 \mathrm{~min}$ at $95{ }^{\circ} \mathrm{C}$ followed by 40 cycles of $95{ }^{\circ} \mathrm{C}$ for $10 \mathrm{~s}$ and $60^{\circ} \mathrm{C}$ for $20 \mathrm{~s}$ was performed. A non-template control was always included in each assay and melting curve analysis was performed at the end of each qPCR to confirm amplification specificity. In addition, amplification products were also checked by agarose gel electrophoresis after each assay.
Fluorescence data obtained after qPCR was analyzed using LinRegPCR, which determines $\mathrm{Cq}$ values and PCR efficiencies [68]. These values were further used to analyze mitochondrial expression; statistical tests were performed using REST 2009 (QIAGEN) as described previously [69].

\section{Additional files}

Additional file 1. Mitochondrial genome of Sinularia cf. cruciata.

Additional file 2. Mitochondrial gene expression determined by RNASeq for Sinularia cf. cruciata, Gorgonia ventalina, and Corallium rubrum.

Additional file 3. Stem-loop structures and conserved motifs of putative control regions from octocorals mitogenomes studied.

Additional file 4. Additional tables (1, 2).

Additional file 5. RHAPA analysis.

Additional file 6. Antisense strand-specific RT-PCR.

Additional file 7. Stem-loop structures and conserved motif of IGRs between detected transcriptional units from S. cf. cruciata.

\section{Abbreviations}

COl: cytochrome oxidase, subunit l; COIl: cytochrome oxidase, subunit Il; ATP6: ATP synthase, subunit 6; ATP8: ATP synthase, subunit 8; ND5: NADH dehydrogenase, subunit 5; ND4: NADH dehydrogenase, subunit 4; ND4L: NADH dehydrogenase, subunit 4L; ND6: NADH dehydrogenase, subunit 6; CytB: cytochrome b; ND1: NADH dehydrogenase, subunit 1; ND2: NADH dehydrogenase, subunit 2; ND3: NADH dehydrogenase, subunit 3;

COIII: cytochrome oxidase, subunit III; 12S: small subunit of mitochondrial ribosomal RNA gene; 16S: large subunit of mitochondrial ribosomal RNA gene.

\section{Authors' contributions}

GGS, SV, and GW conceived and designed the experiments; GGS performed the experiments and analyzed the data; AP helped in bridging the mitogenome sequence gaps and analyzing the genome; GGS drafted the manuscript; and SV and GW helped with its revision. All authors read and approved the final manuscript.

\section{Author details \\ ${ }^{1}$ Department of Earth and Environmental Sciences, Palaeontology \& Geo- biology, Ludwig-Maximilians-Universität München, Richard-Wagner Str. 10, 80333 Munich, Germany. ${ }^{2}$ GeoBio-Center, Ludwig-Maximilians-Universität München (LMU), Richard-Wagner Str. 10, Munich 80333, Germany. ${ }^{3}$ SNSB- Bavarian State Collections of Palaeontology and Geology, Richard-Wagner Str. 10, 80333 Munich, Germany. ${ }^{4}$ Present Address: Marine Biotechnology and Ecology (MBE) Division, CSIR-Central Salt and Marine Chemicals Research Institute (CSMCRI), Bhavnagar, Gujarat 364002, India.}

\section{Acknowledgements}

We are thankful to the editor, the reviewers of the journal, and two anonymous reviewers of Axios Review for insightful feedback on the manuscript. We would like to acknowledge Gabi Büttner and Simone Schätzle for their assistance in the laboratory, Dr. Dirk Erpenbeck and Dr. Oliver Voigt for their constructive discussions, and Dr. Peter Naumann's for his assistance in the aquarium. SV is indebted to M. Vargas Villalobos, S. Vargas Villalobos, S. Vargas Villalobos and N. Villalobos Trigueros for their constant support.

\section{Competing interests}

The authors declare that they have no competing interests.

\section{Availability of data and materials}

The datasets supporting the conclusions of this article are available in GenBank repository (Accession No. KY462727). 


\section{Funding}

The research was funded by DAAD (German Academic Exchange Service) Ph.D. scholarship awarded to GGS. (91540344).

\section{Publisher's Note}

Springer Nature remains neutral with regard to jurisdictional claims in published maps and institutional affiliations.

Received: 11 January 2017 Accepted: 2 June 2017 Published online: 17 June 2017

\section{References}

1. GIGA Community of Scientists. The global invertebrate genomics alliance (GIGA): developing community resources to study diverse invertebrate genomes. J Hered. 2014;105(1):1-18.

2. Bhattacharya D, Agrawal S, Aranda M, Baumgarten S, Belcaid M, Drake JL, Erwin D, Foret S, Gates RD, Gruber DF, et al. Comparative genomics explains the evolutionary success of reef-forming corals. Elife. 2016;5:e13288.

3. Lavrov DV. Key transitions in animal evolution: a mitochondrial DNA perspective. Integr Comp Biol. 2007;47:734-43.

4. Berg OG, Kurland CG. Why mitochondrial genes are most often found in nuclei. Mol Biol Evol. 2000;17:951-61.

5. Adams KL, Palmer JD. Evolution of mitochondrial gene content: gene loss and transfer to the nucleus. Mol Phylogenet Evol. 2003;29(3):380-95.

6. Boore JL. Animal mitochondrial genomes. Nucleic Acids Res. 1999:27(8):1767-80.

7. Lavrov DV, Pett W. Animal mitochondrial DNA as we don't know it: mtgenome organization and evolution in non-bilaterian lineages. Genome Biol Evol. 2016;8(9):2896-913.

8. Rot C, Goldfarb I, Ilan M, Huchon D. Putative cross-kingdom horizontal gene transfer in sponge (Porifera) mitochondria. BMC Evol Biol. 2006;6:71.

9. Erpenbeck D, Aryasari R, Hooper JN, Wörheide G. A mitochondrial intron in a verongid sponge. J Mol Evol. 2015;80(1):13-7.

10. Szitenberg A, Rot C, Ilan M, Huchon D. Diversity of sponge mitochondrial introns revealed by cox 1 sequences of Tetillidae. BMC Evol Biol. 2010;10:288.

11. van Oppen MJ, Catmull J, McDonald BJ, Hislop NR, Hagerman PJ, Miller DJ. The mitochondrial genome of Acropora tenuis (Cnidaria; Scleractinia) contains a large group I intron and a candidate control region. J Mol Evol. 2002;55(1):1-13

12. Huchon D, Szitenberg A, Shefer S, Ilan M, Feldstein T. Mitochondrial group I and group || introns in the sponge orders Agelasida and Axinellida. BMC Evol Biol. 2015:15:278

13. Pont-Kingdon GA, Okada NA, Macfarlane JL, Beagley CT, Wolstenholme DR, Cavalier-Smith T, Clark-Walker GD. A coral mitochondrial mutS gene. Nature. 1995;375:109-11.

14. Flot JF, Tillier S. The mitochondrial genome of Pocillopora (Cnidaria: Scleractinia) contains two variable regions: the putative D-loop and a novel ORF of unknown function. Gene. 2007;401(1-2):80-7.

15. Park E, Song JI, Won YJ. The complete mitochondrial genome of Calicogorgia granulosa (Anthozoa: Octocorallia): potential gene novelty in unidentified ORFs formed by repeat expansion and segmental duplication. Gene. 2011;486(1-2):81-7.

16. Lin MF, Kitahara MV, Luo H, Tracey D, Geller J, Fukami H, Miller DJ, Chen CA. Mitochondrial genome rearrangements in the Scleractinia/Corallimorpharia complex: implications for coral phylogeny. Genome Biol Evol. 2014;6(5):1086-95.

17. Lavrov DV, Pett W, Voigt O, Wörheide G, Forget L, Lang BF, Kayal E. Mitochondrial DNA of Clathrina clathrus (Calcarea, Calcinea): six linear chromosomes, fragmented rRNAs, tRNA editing, and a novel genetic code. Mol Biol Evol. 2013;30(4):865-80

18. Voigt O, Erpenbeck D, Wörheide G. A fragmented metazoan organellar genome: the two mitochondrial chromosomes of Hydra magnipapillata. BMC genom. 2008;9:350.

19. Mercer TR, Neph S, Dinger ME, Crawford J, Smith MA, Shearwood AM, Haugen E, Bracken CP, Rackham O, Stamatoyannopoulos JA, et al. The human mitochondrial transcriptome. Cell. 2011;146(4):645-58.
20. Coucheron DH, Nymark M, Breines R, Karlsen BO, Andreassen M, Jørgensen TE, Moum T, Johansen SD. Characterization of mitochondrial mRNAs in codfish reveals unique features compared to mammals. Curr Genet. 2011;57:213-22.

21. Stewart JB, Beckenbach AT. Characterization of mature mitochondrial transcripts in Drosophila, and the implications for the tRNA punctuation model in arthropods. Gene. 2009:445(1-2):49-57.

22. Markova S, Filipi K, Searle JB, Kotlik P. Mapping $3^{\prime}$ transcript ends in the bank vole (Clethrionomys glareolus) mitochondrial genome with RNA-Seq BMC Genom. 2015;16:870.

23. Temperley RJ, Wydro M, Lightowlers RN, Chrzanowska-Lightowlers ZM. Human mitochondrial mRNAs-like members of all families, similar but different. Biochim Biophys Acta. 2010;1797(6-7):1081-5.

24. Ojala D, Montoya J, Attardi G. tRNA punctuation model of RNA processing in human mitochondria. Nature. 1981;290(5806):470-4.

25. Rossmanith $W$. Of $P$ and $Z$ : mitochondrial tRNA processing enzymes. Biochim Biophys Acta. 2012;1819(9-10):1017-26.

26. Nagaike T, Suzuki T, Ueda T. Polyadenylation in mammalian mitochondria: insights from recent studies. Biochim Biophys Acta. 2008;1779(4):266-9.

27. Slomovic S, Laufer D, Geiger D, Schuster G. Polyadenylation and degradation of human mitochondrial RNA: the prokaryotic past leaves its mark. Mol Cell Biol. 2005;25(15):6427-35.

28. Kayal E, Bentlage B, Cartwright P, Yanagihara AA, Lindsay DJ, Hopcroft RR, Collins AG. Phylogenetic analysis of higher-level relationships within Hydroidolina (Cnidaria: Hydrozoa) using mitochondrial genome data and insight into their mitochondrial transcription. PeerJ. 2015;3:e1403.

29. Kayal E, Bentlage B, Collins AG. Insights into the transcriptional and translational mechanisms of linear organellar chromosomes in the box jellyfish Alatina alata (Cnidaria: Medusozoa: Cubozoa). RNA Biol. 2016;13:1-11.

30. Brugler MR, France SC. The mitochondrial genome of a deep-sea bamboo coral (Cnidaria, Anthozoa, Octocorallia, Isididae): genome structure and putative origins of replication are not conserved among octocorals. J Mol Evol. 2008:67(2):125-36.

31. Brockman SA, McFadden CS. The mitochondrial genome of Paraminabea aldersladei (Cnidaria: Anthozoa: Octocorallia) supports intramolecular recombination as the primary mechanism of gene rearrangement in octocoral mitochondrial genomes. Genome biol evol. 2012;4:994-1006.

32. Uda K, Komeda Y, Koyama H, Koga K, Fujita T, Iwasaki N, Suzuki T. Complete mitochondrial genomes of two Japanese precious corals, Paracorallium japonicum and Corallium konojoi (Cnidaria, Octocorallia, Coralliidae): notable differences in gene arrangement. Gene. 2011;476:27-37.

33. Figueroa DF, Baco AR. Octocoral mitochondrial genomes provide insights into the phylogenetic history of gene order rearrangements, order reversals, and cnidarian phylogenetics. Genome Biol Evol. 2015;7(1):391-409.

34. Pont-Kingdon G, Okada NA, Macfarlane JL, Beagley CT, Watkins-Sims CD, Cavalier-Smith T, Clark-Walker GD, Wolstenholme DR. Mitochondrial DNA of the coral Sarcophyton glaucum contains a gene for a homologue of bacterial MutS: a possible case of gene transfer from the nucleus to the mitochondrion. J Mol Evol. 1998;46:419-31.

35. Bilewitch JP, Degnan SM. A unique horizontal gene transfer event has provided the octocoral mitochondrial genome with an active mismatch repair gene that has potential for an unusual self-contained function. BMC Evol Biol. 2011;11:228.

36. Shearer TL, Van Oppen MJH, Romano SL, Wörheide G. Slow mitochondrial DNA sequence evolution in the Anthozoa (Cnidaria). Mol Ecol. 2002;11:2475-87

37. Hellberg ME. No variation and low synonymous substitution rates in coral mtDNA despite high nuclear variation. BMC Evol Biol. 2006;6:24.

38. Lynch M, Koskella B, Schaack S. Mutation pressure and the evolution of organelle genomic architecture. Science (New York). 2006;311:1727-30.

39. MCFadden CS, Benayahu Y, Pante E, Thoma JN, Nevarez PA, France SC. Limitations of mitochondrial gene barcoding in Octocorallia. Mol ecol resour. 2011;11:19-31.

40. Burge CA, Mouchka ME, Harvell CD, Roberts S. Immune response of the Caribbean sea fan, Gorgonia ventalina, exposed to an Aplanochytrium parasite as revealed by transcriptome sequencing. Front Physiol. 2013:4:180.

41. Pratlong M, Haguenauer A, Chabrol O, Klopp C, Pontarotti P, Aurelle D. The red coral (Corallium rubrum) transcriptome: a new resource for population genetics and local adaptation studies. Mol Ecol Resour. 2015:15(5):1205-15. 
42. Uda K, Komeda Y, Fujita T, Iwasaki N, Bavestrello G, Giovine M, CattaneoVietti R, Suzuki T. Complete mitochondrial genomes of the Japanese pink coral (Corallium elatius) and the Mediterranean red coral (Corallium rubrum): a reevaluation of the phylogeny of the family Coralliidae based on molecular data. Comp Biochem Physiol Part D Genomics Proteom. 2013;8(3):209-19.

43. Rinn JL, Chang HY. Genome regulation by long noncoding RNAs. Annu Rev Biochem. 2012;81:145-66.

44. Gray MW. Origin and evolution of organelle genomes. Curr Opin Genet Dev. 1993;3(6):884-90.

45. Dyall SD, Brown MT, Johnson PJ. Ancient invasions: from endosymbionts to organelles. Science. 2004;304(5668):253-7.

46. Emblem A, Okkenhaug S, Weiss ES, Denver DR, Karlsen BO, Moum T, Johansen SD. Sea anemones possess dynamic mitogenome structures. Mol Phylogenet Evol. 2014;75:184-93.

47. Helfenbein KG, Fourcade HM, Vanjani RG, Boore JL. The mitochondrial genome of Paraspadella goto i is highly reduced and reveals that chaetognaths are a sister group to protostomes. Proc Natl Acad Sci USA 2004;101(29):10639-43.

48. Wang X, Lavrov DV. Seventeen new complete mtDNA sequences reveal extensive mitochondrial genome evolution within the Demospongiae. PLOS ONE. 2008:3:e2723.

49. Beagley CT, Okimoto R, Wolstenholme DR. The mitochondrial genome of the sea anemone Metridium senile (Cnidaria): introns, a paucity of tRNA genes, and a near-standard genetic code. Genetics. 1998;148(3):1091-108.

50. Mignone F, Gissi C, Liuni S, Pesole G. Untranslated regions of mRNAs. Genome Biol. 2002;3(3):REVIEWS0004.

51. Dowton M, Campbell NJ. Intramitochondrial recombination-is it why some mitochondrial genes sleep around? Trends Ecol Evol. 2001;16(6):269-71.

52. Dowton M, Cameron SL, Dowavic Jl, Austin AD, Whiting MF. Characterization of 67 mitochondrial tRNA gene rearrangements in the hymenoptera Suggests that mitochondrial tRNA gene position is selectively neutral. Mol Biol Evol. 2009;26(7):1607-17.

53. Bai Y, Shakeley RM, Attardi G. Tight control of respiration by NADH dehydrogenase ND5 subunit gene expression in mouse mitochondria. Mol Cell Biol. 2000;20(3):805-15.

54. Mohanty BK, Kushner SR. Bacterial/archaeal/organellar polyadenylation. Wiley Interdiscip Rev RNA. 2011;2(2):256-76

55. Obmolova G, Ban C, Hsieh P, Yang W. Crystal structures of mismatch repair protein MutS and its complex with a substrate DNA. Nature. 2000;407(6805):703-10.
56. Lutz CS, Moreira A. Alternative mRNA polyadenylation in eukaryotes: an effective regulator of gene expression. Wiley Interdiscip Rev RNA. 2011;2(1):22-31.

57. Cocquet J, Chong A, Zhang G, Veitia RA. Reverse transcriptase template switching and false alternative transcripts. Genomics. 2006;88:127-31.

58. Mercer TR, Gerhardt DJ, Dinger ME, Crawford J, Trapnell C, Jeddeloh JA, Mattick JS, Rinn JL. Targeted RNA sequencing reveals the deep complexity of the human transcriptome. Nat Biotechnol. 2012;30(1):U99-147.

59. Rackham O, Shearwood AM, Mercer TR, Davies SM, Mattick JS, Filipovska A. Long noncoding RNAs are generated from the mitochondrial genome and regulated by nuclear-encoded proteins. RNA. 2011:17(12):2085-93.

60. Salinas-Giege T, Giege R, Giege P. tRNA biology in mitochondria. Int J Mol Sci. 2015;16(3):4518-59.

61. Haen KM, Pett W, Lavrov DV. Parallel loss of nuclear-encoded mitochondrial aminoacyl-tRNA synthetases and mtDNA-encoded tRNAs in Cnidaria. Mol Biol Evol. 2010;27(10):2216-9.

62. Rackham O, Mercer TR, Filipovska A. The human mitochondrial transcriptome and the RNA-binding proteins that regulate its expression. Wiley Interdiscip Rev RNA. 2012;3(5):675-95

63. Kearse M, Moir R, Wilson A, Stones-Havas S, Cheung M, Sturrock S, Buxton S, Cooper A, Markowitz S, Duran C, et al. Geneious basic: an integrated and extendable desktop software platform for the organization and analysis of sequence data. Bioinformatics. 2012;28(12):1647-9.

64. Slomovic S, Schuster G. Circularized RT-PCR (CRT-PCR): analysis of the $5^{\prime}$ ends, $3^{\prime}$ ends, and poly(A) tails of RNA. Methods Enzymol. 2013;530:227-51.

65. Cornett AL, Lutz CS. RHAPA: a new method to quantify alternative polyadenylation. Methods Mol Biol. 2014;1125:157-67.

66. Ho EC, Donaldson ME, Saville BJ. Detection of antisense RNA transcripts by strand-specific RT-PCR. Methods Mol Biol. 2010;630:125-38.

67. Michel U, Stringaris AK, Nau R, Rieckmann P. Differential expression of sense and antisense transcripts of the mitochondrial DNA region coding for ATPase 6 in fetal and adult porcine brain: identification of novel unusually assembled mitochondrial RNAs. Biochem Biophys Res Commun. 2000;271(1):170-80.

68. Ruijter JM, Ramakers C, Hoogaars WMH, Karlen Y, Bakker O, van den Hoff MJB, Moorman AFM. Amplification efficiency: linking baseline and bias in the analysis of quantitative PCR data. Nucleic Acids Res. 2009;37:e45.

69. Pfaffl MW, Horgan GW, Dempfle L. Relative expression software tool (REST) for group-wise comparison and statistical analysis of relative expression results in real-time PCR. Nucleic Acids Res. 2002;30(9):e36.

\section{Submit your next manuscript to BioMed Central and we will help you at every step:}

- We accept pre-submission inquiries

- Our selector tool helps you to find the most relevant journal

- We provide round the clock customer support

- Convenient online submission

- Thorough peer review

- Inclusion in PubMed and all major indexing services

- Maximum visibility for your research

Submit your manuscript at www.biomedcentral.com/submit
BioMed Central 